\title{
Smart Gas Sensing and Actuation Using Multimode of a MOFs Coated Microbeam
}

\author{
N. Jaber, S. Ilyas, O. Shekhah, M. Eddaoudi, and M. I. Younis* \\ King Abdullah University of Science and Technology, Thuwal 23955-6900, Saudi Arabia \\ *e-mail: mohammad.younis@kaust.edu.sa
}

\begin{abstract}
Smart sensing systems suffers complexity requiring interface circuits, microcontrollers, switches, and actuators to detect and sense, process the signal and take a decision, and trigger an action upon demand. This increases the device footprint and boosts significantly the power required to actuate the system. Here, we present a hybrid sensor and switch device, which is capable of accurately measuring gas concentration and perform switching when the concentration exceeds specific (safe) threshold. The device is based on a clamped-clamped microbeam coated with metalorganic frameworks (MOFs). Using the electrostatic harmonic voltage, we employ dynamic multi-modal actuation in which the microbeam is simultaneously excited at the first mode of vibration, near the pull-in band, and at the third mode. We demonstrate experimentally the effectiveness of this technique in measuring the concentration of water vapor and achieving switching when the concentration exceeds a threshold value. In contrast to a single mode operation, we show that employing multi-modal excitation enhances sensitivity, improves accuracy, and strengthen noise immunity.
\end{abstract}

Keywords-Resonator; Multi-modal; MOFs; Smart sensor; and bifurcation.

\section{INTRODUCTION}

MEMS and NEMS-based resonators have been utilized in detecting minute mass and low gas concentrations [1-3]. These mainly employ electrostatic actuation, which is the most common method due to the unique advantages of low power consumptions and compatibility with CMOS circuits $[4,5]$. Various dynamical principles have been used to enhance sensitivity, such as higher order modes of vibrations $[6,7]$, bifurcation points, and the pull-in instability [8-12]. Recently, the simultaneous actuation and tracking of the frequency shift of multiple vibrational modes of a single resonator has been utilized to enhance the resonator stability [13], reveal the spatial distribution of adsorbed particles [14], measure the mass, position, and velocity of a particle flowing inside a microchannel [1], and also measure the evaporation rate of Polyethylene Glycol [15]. The collective information from different modes of vibrations of a single resonator enable the detection of multiple physical stimuli using a single device. This reduces the power required to operate the sensor and the overall device size, cost, and complexity [1].

In previous works $[10,11]$, we utilized the pull in band near the first mode to enhance the sensitivity of the sensor and perform switching when the concentration exceeds a certain threshold. As higher order modes are known to boost sensitivity in resonant sensors, exciting the resonator near the pull-in bands of the higher order modes should lead in principle to more sensitive switching. However, this requires special designs and impractically high actuation voltages [6]. Also, the robustness and accuracy of the bifurcation based sensing need to be ensured. In close proximity of the bifurcation point, the basin of attraction gets eroded, and the noise effect becomes significant, which reduce the reliability and accuracy of the sensor measurement $[8,11]$.
Despite the previous efforts in realizing smart sensors and switches based on the first mode, the low sensitivity of the first mode and the reduced accuracy near the bifurcation point remains main issues. To resolve these issues, we demonstrate here the employment of two modes of vibration of a clamped-clamped microbeam coated with MOFs and operated in air to selectively detect and accurately measure the concentration of particular gas and perform switching, at reduced voltage, when the concentration exceeds beyond a desired value. As a case study, we utilize the device to sense water vapor concentration and perform switching when the concentration exceeds a selected threshold value.

\section{FABRICATION}

The proposed device is based on a clamped-clamped microbeam resonator functionalized with metal organic frame works (MOFs) for sensitive and selective detection [16-19]. The resonator is electrostatically actuated using twothird lower electrode configuration.

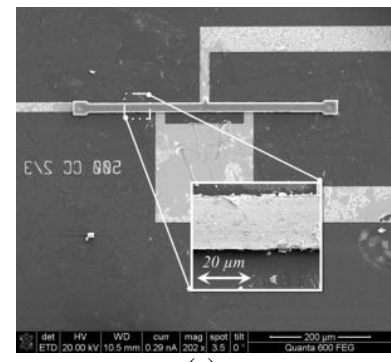

(a)

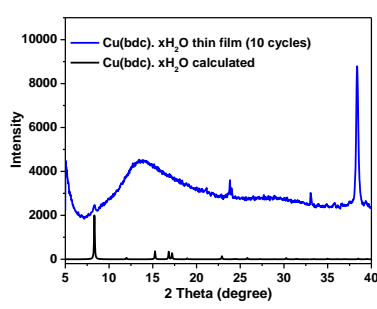

(b)

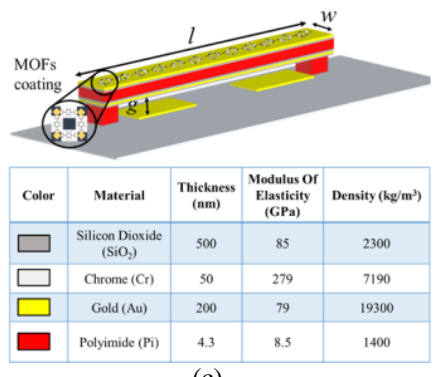

(c)

Fig. 1. (a) An SEM image of the fabricated microbeam with MOFs functionalization. (b) XRD pattern of $\mathrm{Cu}(\mathrm{bdc}) \cdot \mathrm{xH}_{2} \mathrm{O}$ thin film on microbeam (blue) and calculated (black). (c) Schematic of the microbeam with the twothird lower electrode showing the material types, properties, and thicknesses.

In previous work [6], we showed that the two-third configuration reduces the voltage values required to excite the third mode of vibration to one-third compared with the full electrode configuration. The details of the fabrication process can be found in [12]. Figures 1a-1c show an SEM image of the clamped-clamped resonator, the XRD pattern confirmed the formation of the targeted MOF thin film on the microbeam, and a schematic that demonstrates the microbeam design and dimensions; length (l) $500 \mu \mathrm{m}$, width (w) $20 \mu \mathrm{m}$, and gap (g) $3.3 \mu \mathrm{m}$. These dimensions are selected to minimize the squeeze film damping in accordance with the fabrication process rules [20]. 


\section{EXPERIMENTAL SETUP}

We utilize a laser Doppler vibrometer to measure the resonance frequency values and their corresponding mode shapes, record the frequency response curves, and track the change in the amplitude of vibration due to water vapor exposure. A data acquisition card (DAQ) connected to an amplifier is used to actuate the microbeam with a wide range of frequencies and voltage amplitudes. Using the LabVIEW software, the measured real-time data are post-processed to generate the frequency response curves and the amplitude shift due to water vapor exposure. The microbeam is placed inside the test chamber, which is equipped with ports to provide the actuation signals. The water vapor is generated by flowing the nitrogen inside a bubbler placed in a controlled temperature bath. The vapor concentration is governed by controlling the flow of the dry nitrogen line and bubbler line. The experimental setup is shown in Fig. 2.

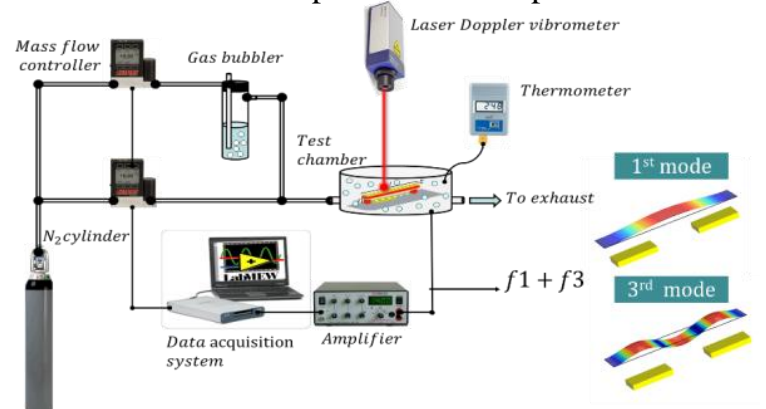

Fig. 2. Schematic of the experimental setup used for testing the device

$$
\text { IV. RESULTS }
$$

\section{A. Frequency response curves}

To experimentally characterize the microbeam, we actuate the microbeam with a harmonic AC signal superimposed to a DC voltage, where the frequency of the AC signal is swept around the mode of interest while using the laser to monitor the microbeam amplitude at the midpoint of the microbeam. The frequency response curves are generated by taking the maximum steady state amplitude $W_{\max }$ at each frequency step. Figure $3 \mathrm{a}$ and $3 \mathrm{~b}$ show the results for $V_{D C}=30 \mathrm{~V}$ and at atmospheric pressure near the first and third modes for different $V_{A C}$ values. The reported results help in selecting the appropriate actuating voltages to obtain a large signal-tonoise ratio. Also, we employ the frequency response curve in converting the measured amplitude change due to water vapor adsorption into frequency values.

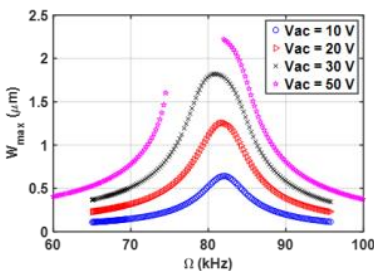

(a)

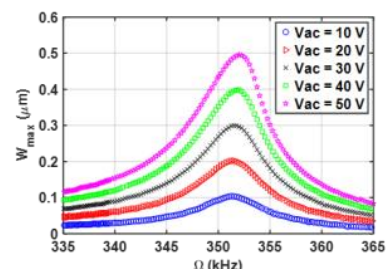

(b)
Fig. 3. Response of the microbeam at $V_{D C}=30 \mathrm{~V}$ and at atmospheric chamber pressure for different AC voltages (a) near the first mode and (b) near the third mode. The open curve in (a) shows a pull-in band.

B. Sensitivity

Before exposing the device to water vapor, we flush the testing chamber with Nitrogen at a flow rate of $2 \mathrm{~L} / \mathrm{min}$ for extended period of time. Then, we expose the resonator to water vapor while monitoring the change in the amplitude of vibration. In our experiments, we fix the operating points at $75 \mathrm{kHz}$ near the first mode and $344 \mathrm{kHz}$, near the third mode.
As water molecules get adsorbed on the device surface, the amplitude of vibration at the selected operating points increases following the left branch of the frequency response curves. The water vapor concentration is controlled by controlling the flow ratio between the bubbler line and the dry nitrogen line. The vapor concentration values are calculated following the procedure demonstrated in [12]. The repeatability is demonstrated by replacing the water vapor flow with nitrogen to flush the resonator and return to the original state; this process is repeated for two cycles as shown in Fig 4a. Figure 4b shows the device response to the different levels of water vapor concentrations. The frequency shift rises linearly as we increase the water vapor concentration, Fig. 4c. Also, we ensured the reversibility by completely flushing the device after each exposure cycle. The experimentally calculated responsivity near the first mode and third mode demonstrate the enhanced sensitivity near the higher order modes of vibration.

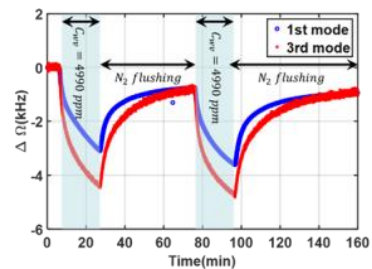

(a)

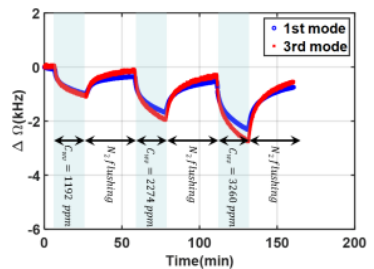

(b)

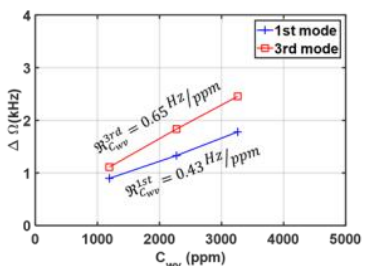

(c)

Fig. 4. Real time measurement of the frequency shift near the first $(75 \mathrm{kHz})$ and third $(344 \mathrm{kHz})$ mode due to vapor exposures. (a) Repeatability of the response at fixed water vapor concentration $C_{w v}=4990 \mathrm{ppm}$. (b) Linearity of the response at different water vapor concentrations. (c) Experimentally calculated sensor responsivity near the first and third modes, which demonstrates the enhanced sensitivity near the higher order mode of vibration.

\section{Multi-modal smart switching}

It is desirable to realize a smart switch that can be utilized to perform an action when the gas concentration exceeds a certain threshold while accurately measuring the concentration. Although, the concept can be employed in principle to detect any type of harmful gas, in our experiment we utilize water vapor for safety reasons. The idea is based on actuating the device with a harmonic signal that has two frequencies $\Omega_{1}$ and $\Omega_{2}$ with $\mathrm{AC}$ amplitudes $V_{A C l}$ and $V_{A C 2}$ superimposed to a DC voltage. $V_{A C l}$ is selected such that the frequency response near the first mode shows a nonlinear response with dynamic pull-in band, Fig. 5a, while $\Omega_{l}$ is tuned close to the dynamic pull-in band of the first mode. The difference between $\Omega_{l}$ and the bifurcation frequency defines the threshold concentration value after which the microbeam collapses due to dynamic pull-in, hence, can be realized as an electrical switch. $V_{A C 2}$ is chosen such that a linear response is realized near the third mode as shown in Fig. 5b. $\Omega_{2}$ is set to be close to the third mode of vibration. The advantage of measuring the shift in the frequency of the third mode that it provides an independent, noise tolerant, and sensitive probe for 
accurately measuring the gas concentration compared to the first mode bifurcation-based sensing, which is less sensitive and more affected by noise thereby dramatically reducing the accuracy of the sensor readings [11].

Next, we illustrate the mass loading experiment. As water molecules get adsorbed on the resonator, the resonance frequencies decrease, and the corresponding amplitudes at each excitation frequency increase following the left branch of the frequency response curves shown in Fig. 5a and Fig. 5b. As a case study, a vapor concentration of $3260 \mathrm{ppm}$ is considered as the threshold value. As shown in Fig. 4c, when operating the resonator in the linear regime, a concentration of $3260 \mathrm{ppm}$ shifts the first mode frequency by $1.78 \mathrm{kHz}$ and the third mode by $2.455 \mathrm{kHz}$. To experimentally demonstrate the concept of smart switching, we fix the first source frequency at $\Omega_{I}=68 \mathrm{kHz}$ (first mode) and voltage amplitude $V_{A C l}=50 \mathrm{~V}$, the second source frequency at $\Omega_{2}=344 \mathrm{kHz}$ (third mode) and voltage amplitude $V_{A C 2}=40 \mathrm{~V}$, and use $V_{D C}=30 \mathrm{~V}$. Ruzziconi et al. [21] demonstrated that the stability of the resonator at a particular operating frequency depends on the size of the safe basin of attraction. As the driving frequency approaches the bifurcation frequency, the safe basin of attraction gets eroded and shrinks in size. To ensure the stability of the resonator at the operating points, we monitor the amplitude fluctuation over time due to noise as shown in the inset of Fig. 5d. The calculated Allan deviation near the first and third mode of vibrations are $\sigma_{f 1}(100)=388 \mathrm{ppm}$ and $\sigma_{f 3}(100)=6 \mathrm{ppm} \quad$, which correspond to frequency fluctuation of $\Delta f_{1}=26 \mathrm{~Hz}$ and $\Delta f_{3}=2 \mathrm{~Hz}$. Hence, the minimum detectable water vapor concentration is $3 \mathrm{ppm}$. Then, the microbeam is exposed to water vapor at the threshold concentration of $3260 \mathrm{ppm}$. As shown in Fig. 5c and Fig. 5d, when the frequency shift near the first mode exceeds the threshold value $(\sim 1.8 \mathrm{kHz})$, the upper electrode collapses into the lower electrode (simulating a switching action). However, the measured frequency shift near the third mode is $1.6 \mathrm{kHz}$, which is less than the expected value at this concentration, $2.455 \mathrm{kHz}$, reported in Fig. 4c. In the vicinity of the bifurcation frequency, the noise influence becomes significant, hence, the measured frequency shift near the first mode can be attributed to the combined effect of noise and water adsorption on the resonator surface $[8,11]$. In our case study, although the threshold concentration is set at 3260 ppm, the measured frequency shift near the third mode indicates that the switching occurred at $1924 \mathrm{ppm}$, Fig. 5d. This proves the importance of using the third mode as an independent channel of information to accurately measure the concentration at which pull in occurs.

The reported results show the significance of this technique in accurately measuring the concentration of water vapor while still being able to switch if the concentration exceeds beyond a certain safe value. The reported noise affect can be used as safety factor, which ensures that the switching action will take place at a gas concentration equal or less than the estimated threshold value based on the idealized curve (based on slow sweeping) of Fig. 5a. Analytically, the robustness of bifurcation based gas sensors can be investigated by conducting a global dynamics analysis on the stable solution before pull-in, which can be used to identify the range of stable solutions. In contrast to previous works using a single mode to demonstrate smart sensor, where the noise effect in the vicinity of the bifurcation frequency is significant reducing the accuracy of the measurement, we show a noise immune technique that can accurately measure the gas concentration by utilizing the higher order modes of vibrations of the same resonators, while still being able to switch upon exceeding a safe concentration value. Also, utilizing the third mode improves the sensor sensitivity and resolution. The reported findings can be easily extended to measure the change in other physical stimuli, such as temperature, and switch upon exceeding the critical limit. Furthermore, employing higher number of modes opens the door for simultaneous measurement of multiple physical parameters using the same resonator, which reduces the power consumption, fabrication cost, and size of the sensor.

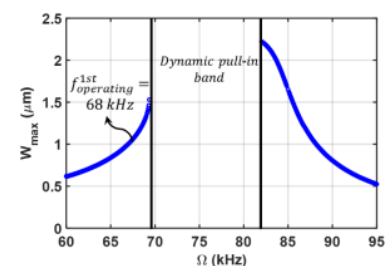

(a)

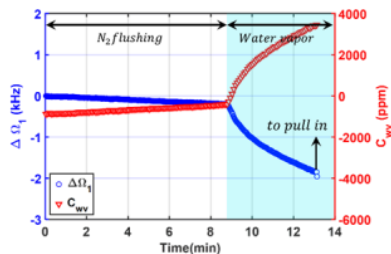

(c)

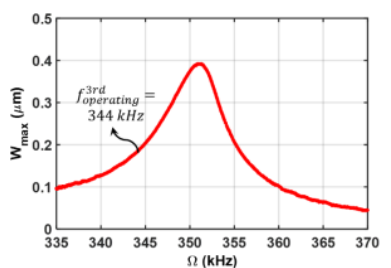

(b)

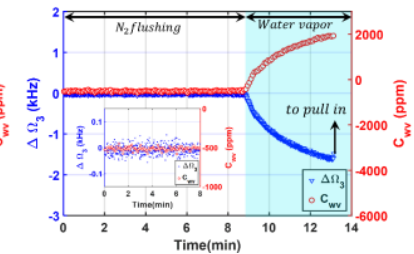

(d)
Fig. 5 Frequency response curves at $V_{D C}=30 \mathrm{~V}$ and at atmospheric pressure (a) near the first mode at $V_{A C l}=50 \mathrm{~V}$ showing the softening behavior and the dynamic pull-in band, (b) near the third mode at $V_{A C 2}=40$ $V$. Real time measurement of the frequency change and the watervapor concentration until pull in. The vapor exposure started at time $=9$ minutes. (c) Near the first mode, (d) near the third mode. Inset: the fluctuation due to noise.

\section{CONCLUSIONS}

In conclusion, we demonstrated a smart sensor that is capable of accurately measuring the concentration of a particular gas and perform switching upon exceeding a certain threshold value. This is in contrast to a single mode operation, in which the noise effect in the vicinity of the bifurcation reduces the accuracy and reliability of the sensor readings. The method is based on simultaneously actuating the resonator near the pull-in band of the first mode and the linear response of the third mode. As a case study, we utilized this concept to measure the environmental humidity. A minimum detectable water vapor concentration of $3 \mathrm{ppm}$ is reported near the third mode of vibration. This concept can be easily employed to detect more harmful gases by functionalizing the microbeam with a selective coating material. Also, it can be utilized and extended to smartly independently detect other physical stimuli, such as temperature. Optimizing the resonator design to excite higher order modes will open the door for simultaneously measuring multiple physical stimuli using a single resonator, which reduces the power consumption, device size, and cost. 


\section{REFERENCES}

[1] Olcum, S., Cermak, N., Wasserman, S. C. \& Manalis, S. R. High-speed multiple-mode mass-sensing resolves dynamic nanoscale mass distributions. Nature Communications 6, 7070, (2015).

[2] Urbiztondo, M., Pellejero, I., Villarroya, M., Sesé, J., Pina, M. P., Dufour, I., and Santamaria, J. Zeolite-modified cantilevers for the sensing of nitrotoluene vapors. Sensors and Actuators B: Chemical, 137(2), pp. 608-616 (2009).

[3] Forsen, E., Abadal, G., Ghatnekar-Nilsson, S., Teva, J., Verd, J., Sandberg, R., Svendsen, W., Perez-Murano, F., Esteve, J., and Figueras, E. Ultrasensitive mass sensor fully integrated with complementary metal-oxide-semiconductor circuitry. Applied Physics Letters, 87(4), p. 043507 (2005)

[4] Sam, J., Kumar, J., Tetteh, E. A., and Braineard, E. P. A study of why electrostatic actuation is preferred and a simulation of an electrostatically actuated cantilever beam for mems applications. International Journal of Engineering Sciences \& Emerging Technologies, 6(5), pp. 441-446 (2014).

[5] Younis, M. I. MEMS Linear and Nonlinear Statics and Dynamics, (Springer, 2011)

[6] Jaber, N., Ramini, A., Carreno, A. A., and Younis, M. I. Higher order modes excitation of electrostatically actuated clampedclamped microbeams: experimental and analytical investigation. Journal of Micromechanics and Microengineering, 26(2), p. 025008 (2016).

[7] Dohn, S., Sandberg, R., Svendsen, W., and Boisen, A. Enhanced functionality of cantilever based mass sensors using higher modes. Applied Physics Letters, 86(23), p. 233501 (2005).

[8] Nguyen, V.-N., Baguet, S., Lamarque, C.-H., and Dufour, R. Bifurcation-based micro-/nanoelectromechanical mass detection. Nonlinear Dynamics, 79(1), pp. 647-662 (2015)

[9] Kumar, V., Boley, J. W., Yang, Y., Ekowaluyo, H., Miller, J. K., Chiu, G. T.-C., and Rhoads, J. F. Bifurcation-based mass sensing using piezoelectrically-actuated microcantilevers. Applied Physics Letters, 98(15), p. 153510 (2011).

[10] Bouchaala, A., Jaber, N., Shekhah, O., Chernikova, V., Eddaoudi, M., and Younis, M. I. A smart microelectromechanical sensor and switch triggered by gas. Applied Physics Letters, 109(1), p. 013502 (2016).
[11] Younis, M. I. \& Alsaleem, F. Exploration of New Concepts for Mass Detection in Electrostatically-Actuated Structures Based on Nonlinear Phenomena. Journal of Computational and Nonlinear Dynamics 4, 21010-21015 (2009).

[12] Jaber, N., Ilyas, S., Shekhah, O., Eddaoudi, M. \& Younis, M. I. Resonant Gas Sensor and Switch Operating in Air With MetalOrganic Frameworks Coating. Journal of Microelectromechanical Systems 27, 156-163 (2018)

[13] Antonio, D., Zanette, D. H. \& López, D. Frequency stabilization in nonlinear micromechanical oscillators. Nature Communications 3, 806, (2012).

[14] Hanay, M. S. et al. Single-protein nanomechanical mass spectrometry in real time. Nature Nanotechnology 7, 602-608 (2012).

[15] Prasad, A. \& Seshia, A. A. Detection of phase transition in polyethylene glycol using a multimodal micromechanical acoustic resonator. Applied Physics Letters 110, 134101, (2017).

[16] Yassine, O. et al. H2S Sensors: Fumarate-Based fcu-MOF Thin Film Grown on a Capacitive Interdigitated Electrode. Angewandte Chemie International Edition 55, 15879-15883 (2016).

[17] Yim, C. et al. CO2-Selective Nanoporous Metal-Organic Framework Microcantilevers. Scientific Reports 5, 10674 (2015).

[18] Yamagiwa, H. et al. Detection of Volatile Organic Compounds by Weight-Detectable Sensors coated with Metal-Organic Frameworks. Scientific Reports 4, 6247-6251 (2014).

[19] Sapsanis, C. et al. Insights on Capacitive Interdigitated Electrodes Coated with MOF Thin Films: Humidity and VOCs Sensing as a Case Study. Sensors 15, 18153-18166 (2015).

[20] Arevalo, A. et al. A versatile multi-user polyimide surface micromachinning process for MEMS applications in IEEE 10th International Conference on Nano/Micro Engineered and Molecular Systems (NEMS),561-565 (2015)

[21] Ruzziconi, L., Ramini, A., Younis, M. \& Lenci, S. Theoretical Prediction of Experimental Jump and Pull-In Dynamics in a MEMS Sensor. Sensors 14, 17089 (2014). 